\title{
Solvent-free synthesis of a series of differently $N$-substituted 4-amino-2-methylquinazolines under microwave irradiation
}

\author{
Vanya B. Kurteva,* Vessela N. Zlatanova, and Vladimir D. Dimitrov \\ Institute of Organic Chemistry, Bulgarian Academy of Sciences, Acad. G. Bonchev str., bl. 9, \\ 1113 Sofia, Bulgaria \\ E-mail:vkurteva@orgchm.bas.bg
}

\begin{abstract}
A fast, highly efficient and environmentally friendly microwave accelerated solvent-free procedure for the synthesis of a series of differently $\mathrm{N}$-substituted 4-amino-2-methylquinazolines is developed. The transformation proceeded very clean, without any traces of side products. It was shown that aliphatic amines react quantitatively after $5 \mathrm{~min}$ irradiation, while the rate of conversion by anilines strongly depends on the place and the type of the substituents in their molecules. The results are explained by the competition of the steric and electronic effects of the amine substituents. The by-product of the reaction, hydrogen chloride, is quantitatively quenched as ammonium salts by the amines, used as nucleophiles, thus preventing its release into the environment.
\end{abstract}

Keywords: 4-Aminoquinazoline, microwaves, solventless, steric hindrance, electronic effects

\section{Introduction}

Quinazolines are a big family of heterocyclic compounds, which have shown broad variety of biological activity profiles, ${ }^{1,2}$ such as analgesic, narcotic, diuretic, antihypertensive, antimalarial, sedative, hypoglycaemic, antibiotic, antitumoral and many others. It has been found ${ }^{3}$ that the biological activity strongly depends on the type and the place of the substituents in their molecules. Out of the wide substitution patterns known, 4-aminoquinazolines are useful as fungicides, ${ }^{4,5}$ antiinflammatory, ${ }^{6,7}$ anticancer, ${ }^{8,9}$ anti-microbial and anti-hypertensive agents. ${ }^{10,11}$ Some 4-anilinoquinazolines have found to be potential and highly selective inhibitors of human immunoglobulin $\mathrm{E}^{12}$ and epidermal growth factor receptor tyrosine kinase, ${ }^{13}$ which regulates the cell growth and proliferation, so they work as potent antiallergic or anticancer agents, respectively.

Among the broad synthetic pathways for aminoquinazoline preparation ${ }^{14,15}$ the substitution of the chlorine atom in 4-chloroquinazolines by amines seems to be the shortest and the cheapest 
one. The transformation affords also an opportunity to prepare a great number of products due to the wide variety of commercially available amines and anilines.

Microwave assisted reactions have become an established tool in organic synthesis, since they achieve rate enhancement, higher yields and better selectivity in respect to the conventional

heating. ${ }^{16-21}$ The efficient, clean and economic solventless technique, ${ }^{22-24}$ which avoids hazards of solution phase reactions, where high pressures are created in microwave oven at elevated temperatures, is an environmentally benign condition preventing release of reaction products into the environment. Notwithstanding the broad applicability of microwave methodology in the synthesis of heterocyclic compounds, ${ }^{25-29}$ to the best of our knowledge only two papers report the preparation of 4-aminoquinazolines under microwaves, ${ }^{30,31}$ both based on ring-closure reactions of substituted benzonitriles.

A solventless synthesis of a series of differently $N$-substituted 4-amino-2methylquinazolines from 4-chloro-2-methylquinazoline under microwave irradiation (MWI) is presented herein.

\section{Results and Discussion}

The starting 4-chloro-2-methylquinazolin (1) was prepared according to a known procedure ${ }^{32}$ from commercially available 2-methylquinazolin-4(3H)-on. Its conversion into 4aminoquinazolines 2-22 was performed under microwave irradiation in solvent-free conditions, as shown on Scheme 1. Mixtures of chloride $\mathbf{1}$ and an amine were irradiated in a domestic household microwave oven in open vessels with a power of $800 \mathrm{~W}$ to afford the corresponding aminoquinazolines (2-22) in variable rates. The products were isolated by trituration of the crude reaction mixtures with acetone followed by washing with water to remove the ammonium salts formed. Acetone was used because of the better type of the crystals obtained compared to those formed in other organic solvents. When using ether or dichloromethane, for example, pure products were obtained again but in a form of very fine powder, which makes their filtration difficult. A simple water treatment is an explicit work-up itself only in the case of complete reaction, i.e. when the water insoluble starting chloride $\mathbf{1}$ does not exist in the crude reaction mixture. The proposed work-up is fast, easy and clean and presents an advantage of the method independently of the partial solubility of some of the products in acetone, leading to lower isolated yields than the real ones in these cases.

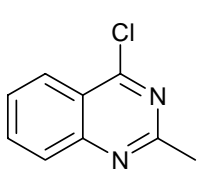

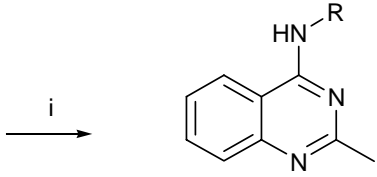

$2-22$

Scheme 1. Synthesis of $N$-substituted 4-amino-2-methylquinazolines 2-22. i) $\mathrm{RNH}_{2}$ (2 equiv), MWI, $800 \mathrm{~W}$. 
The transformation proceeded very clean, without any traces of side products apart from the main one and staring chloride. The by-product of the reaction, hydrogen chloride, was quenched as ammonium salts by the amines, used as nucleophiles. The salts were recovered from the water solutions by removing the solvent and additional drying in vacuo. The quantitative yields obtained are an indication that the harmful by-product is quantitatively quenched in the course of the reaction and thus, avoiding its release into the environment.

The free amines can be recovered by ether-aq. base $\left(\mathrm{NaHCO}_{3}, \mathrm{Na}_{2} \mathrm{CO}_{3}\right.$ etc.) extraction of the water liquors followed by drying and evaporation. However, the manipulation is time and energy consuming and due to the relatively low price of the amines used their recovering is not reasonable in $1 \mathrm{mmol}$ scale but should be of practical importance in scale-up experiments.

The results, which are summarised on Table 1, show that quantitative conversions were achieved after 5-15 min irradiation in the most part of the cases, while a time scale of 5 min could give a qualitative indication about the competitive influence of the aniline substituents on the rate of the transformation studied.

Quantitative conversion was observed in 5 min when aliphatic amines were used (21 and 22). However, the isolation yields were much lower than the real ones, mainly in the case of $N$ benzyl derivative 21, due to the partial solubility of the products in acetone, which can be avoided by applying preparative TLC for their isolation.

In contrast to the aliphatic amines, the anilines used showed different rates of conversion (Table 1) depending on the type and the place of the substituents in their molecules. These results were expected as it is well known that the aliphatic amines are more basic than the aromatic and thus, our attention was focused mainly on the reaction of 4-chloro-2methylquinazoline 1 with differently substituted aromatic amines.

Similar reaction rates were observed when amines of simple aromatic type were used, 2, 19 and 20, while the presence of electrowithdrawing or electrodonating groups considerably affects the transformation. 
Table 1. Synthesis of 4-amino-2-methylquinazolines (2-22) from 4-chloro-2-methylquinazoline (1) under microwave irradiation, $800 \mathrm{~W}$

\begin{tabular}{|c|c|c|c|c|c|c|c|}
\hline \multicolumn{2}{|c|}{ Product } & \multirow{2}{*}{$\begin{array}{l}\text { Reaction } \\
\text { time, min }\end{array}$} & \multirow[t]{2}{*}{ Yield, ${ }^{\mathrm{a}} \%$} & \multicolumn{2}{|c|}{ Product } & \multirow{2}{*}{$\begin{array}{l}\text { Reaction } \\
\text { time, min }\end{array}$} & \multirow[t]{2}{*}{ Yield, ${ }^{\mathrm{a}} \%$} \\
\hline No. & Structure & & & No. & Structure & & \\
\hline \multirow[t]{3}{*}{2} & & 5 & 58 & 13 & & 5 & 62 \\
\hline & & 10 & 86 & & & 10 & 75 \\
\hline & & & & & & 15 & 96 \\
\hline \multirow[t]{3}{*}{3} & & 5 & 55 & 14 & & 5 & 58 \\
\hline & & 10 & 84 & & & 10 & 76 \\
\hline & & & & & & 15 & 99 \\
\hline \multirow[t]{3}{*}{4} & & 5 & 56 & 15 & & 5 & 55 \\
\hline & & 10 & 85 & & & 10 & 77 \\
\hline & & & & & & 15 & 97 \\
\hline \multirow[t]{3}{*}{5} & & 5 & 65 & 16 & & 5 & 42 \\
\hline & & 10 & 93 & & & 10 & 61 \\
\hline & & & & & & 15 & 82 \\
\hline \multirow[t]{3}{*}{6} & & 5 & 42 & 17 & & 10 & 6 \\
\hline & & 10 & 60 & & & 30 & 18 \\
\hline & & 15 & 92 & & & & \\
\hline \multirow[t]{2}{*}{7} & & 5 & 64 & 18 & & 30 & - \\
\hline & & 10 & 94 & & & & \\
\hline \multirow[t]{2}{*}{8} & & 5 & 55 & 19 & & 5 & 79 \\
\hline & & 10 & 91 & & & 10 & 99 \\
\hline \multirow[t]{2}{*}{9} & & 5 & 50 & 20 & & 5 & 73 \\
\hline & & 10 & 83 & & & 10 & 98 \\
\hline 10 & & 5 & 98 & & & & \\
\hline \multirow[t]{2}{*}{11} & & 5 & 67 & 21 & & 5 & $48^{\mathrm{b}}$ \\
\hline & & 10 & 96 & & & & \\
\hline \multirow[t]{2}{*}{12} & & 5 & 63 & 22 & & 5 & $73^{b}$ \\
\hline & & 10 & 94 & & & & \\
\hline
\end{tabular}

${ }^{\mathrm{a}}$ Isolated yield; ${ }^{\mathrm{b}}$ Quantitative conversion. 
Several anilines with electropositive or electronegative substituents were used as reagents and, based on the observed reaction rates, some conclusions and assumptions on the competitive role of the electronic and steric factors could be done. As can be seen on Table 1, the presence of a single substituent in the aniline molecule does not influence the reaction rate significantly. On the contrary, when using di- or tri-substituted anilines, a substantial deviation from the general pattern was observed. Thus, while the product 10, having three electrondonating methoxyl groups, was formed much faster than unsubstituted and mono-substituted compounds, aminoquinolines with two or three electrowithdrawing groups (16-18) were generated with a serious retardation. The influence of the steric hindrance in the reagent molecules on the reaction rates is the most clearly revealed by the slower formation of 2,6-dichloroquinazoline than 2,3analogue, 17 vs 16, as well as by the lower yields of 6 after 5 and 10 min irradiation in comparison with those of o-mono-methyl derivative 5. However, a comparison between 2,6dimethylamino and 2,6-dichloro compounds, 6 vs 17, shows that the methyl groups which are bulkier than chlorine atoms lead to much lower reaction retardation. The latter is in contrast with the stereochemical expectations but can be explained by the influence of the electronic effects of the substituents on the reaction rates, as an electrodonating methyl group affords acceleration, while an electrowithdrawing chlorine atom leads to the opposite effect on the amine reactivity as a norm. This influence is confirmed by the lack of reactivity of 2,4,6-trichloro aniline in the transformation studied in comparison with its equivalently hindered 2,6-dichloro analogue, no formation of 18 after $30 \mathrm{~min} v s \quad 6 \%$ of 17 in $10 \mathrm{~min}$ and $18 \%$ in $30 \mathrm{~min}$. The role of the electronic effects of the aniline substituents is also clearly demonstrated by the fast formation of the trimethoxyquinazoline 10, where complete conversion was achieved in $5 \mathrm{~min}$. Based on these observations it could be suggested that both steric and electronic factors affect the rate of the reaction but the latter seem to be the key ones, $\mathbf{6}$ vs 17 and 17 vs 18.

\section{Conclusions}

A series of differently $N$-substituted 4-amino-2-methylquinazolines was prepared by applying a fast, highly efficient and environmentally friendly solvent-free procedure under microwave irradiation. The transformation proceeded very clean, without any traces of side products apart from the main one and starting chloride. It was shown that aliphatic amines react quantitatively after 5 min irradiation, while the rate of conversion by anilines strongly depends on the place and the type of the substituents in their molecules. The results are explained by the competition of the steric and electronic effects of the aniline substituents and it was found that both factors affect significantly the rate of the transformation studied. As the by-product of the reaction, hydrogen chloride, is quenched as an ammonium salt in the course of the reaction, avoiding its liberation into the air, the method presents itself an additional environmentally benign synthetic advantage. 


\section{Experimental Section}

General Procedures. All reagents were purchased from Aldrich, Merck and Fluka and were used without any further purification. The microwave irradiated reactions (MWI) were performed in domestic household oven Samsung M1777N. Fluka silica gel/TLC-cards 60778 with fluorescent indicator $254 \mathrm{~nm}$ were used for TLC chromatography and $\mathrm{R}_{\mathrm{f}}$-values determination. The melting points were determined in capillary tubes without corrections. The NMR spectra were recorded on a Bruker AVANCE DRX 250 spectrometer in deuteromethanol, the chemical shifts were quoted in ppm in $\delta$-value against tetramethylsilane (TMS) as internal standard and the coupling constants were calculated in $\mathrm{Hz}$. The microanalyses were carried out by the microanalyses service of the Institute of Organic Chemistry, Bulgarian Academy of Sciences.

$\mathrm{N}$-Substituted 4-amino-2-methyl-quinazolines. General procedure. To 4-chloro-2methylquinazoline $1(1 \mathrm{mmol}, 160 \mathrm{mg})$ an amine $(2 \mathrm{mmol})$ was added and the mixture was heated in a MW oven at $800 \mathrm{~W}$ for a period of 5-30 min in consecutive 5 min intervals. To a crude reaction mixture acetone $(5 \mathrm{ml})$ was added and the precipitate formed was filtered off and washed with water. The ammonium salts were recovered in quantitative yields from the water solutions by removing the solvent and additional drying in vacuo. The reaction yields are summarised on Table 1.

2-Methyl- $N$-phenylquinazolin-4-amine (2). ${ }^{33,34}$ m. p. $175-178{ }^{\circ} \mathrm{C}$ (lit. $161-162.5{ }^{\circ} \mathrm{C},{ }^{33} 163$ $165^{\circ} \mathrm{C}^{34}$ ); $\mathrm{R}_{\mathrm{f}} 0.39$ (ethylacetate/hexane 1:1), $\mathrm{R}_{\mathrm{f}} 0.24$ (ethylacetate/hexane $1: 2$ ); ${ }^{1} \mathrm{H}$ NMR 2.674 (s, 3H, $\left.\mathrm{CH}_{3}-2\right), 7.341$ (m, 1H, CH-4'), 7.486 (m, 2H, CH-3' and $\left.\mathrm{CH}-5^{\prime}\right), 7.764(\mathrm{~m}, 4 \mathrm{H}, \mathrm{CH}-5, \mathrm{CH}-$ 7, $\mathrm{CH}-2$ ' and $\mathrm{CH}-6^{\prime}$ ), 8.055 (ddd, $1 \mathrm{H}, \mathrm{J}$ 8.2, 7.3, 1.2, $\mathrm{CH}-6$ ), 8.579 (dd, $1 \mathrm{H}, \mathrm{J} 7.5,1.4, \mathrm{CH}-8$ ); ${ }^{13} \mathrm{C}$ NMR $22.90\left(\mathrm{CH}_{3}-2\right), 113.65$ (C-4a), $117.82\left(\mathrm{CH}-4{ }^{\prime}\right), 120.56(\mathrm{CH}-5), 125.06(\mathrm{CH}-6), 125.69$ ( $\mathrm{CH}-2$ ' and $\left.\mathrm{CH}^{\prime} 6^{\prime}\right), 129.59$ (CH-8), $130.02\left(\mathrm{CH}-3^{\prime}\right.$ and $\mathrm{CH}-5$ '), $137.31(\mathrm{CH}-7), 138.04\left(\mathrm{C}_{\text {quat }}\right)$, 141.33 (C-8a), $161.24(C-2), 163.64(C-4)$; Anal. calc. C76.57, H 5.57, for $\mathrm{C}_{15} \mathrm{H}_{13} \mathrm{~N}_{3}$, found $\mathrm{C}$ 76.72, H 5.61.

2-Methyl- $\boldsymbol{N}$-p-tolylquinazolin-4-amine (3). m. p. $265-268{ }^{\circ} \mathrm{C} ; \mathrm{R}_{\mathrm{f}} 0.39$ (ethylacetate/hexane 1:1), $\mathrm{R}_{\mathrm{f}} 0.24$ (ethylacetate/hexane 1:2); ${ }^{1} \mathrm{H}$ NMR 2.390 (s, $3 \mathrm{H}, \mathrm{CH}_{3}-4^{\prime}$ ), 2.652 (s, $3 \mathrm{H}, \mathrm{CH}_{3}-2$ ), 7.280 (d, 2H, J 8.1, $\mathrm{CH}-3^{\prime}$ ' and $\left.\mathrm{CH}-5^{\prime}\right), 7.618$ (d, 2H, J 8.1, $\mathrm{CH}-2^{\prime}$ ' and $\left.\mathrm{CH}-6^{\prime}\right), 7.765$ (m, 2H, $\mathrm{CH}-5$ and $\mathrm{CH}-7$ ), 8.018 (ddd, $1 \mathrm{H}, \mathrm{J}$ 8.3, 7.3, 1.3, $\mathrm{CH}-6), 8.535$ (dd, $1 \mathrm{H}, \mathrm{J} 8.5,1.3, \mathrm{CH}-8) ;{ }^{13} \mathrm{C}$ NMR $21.16\left(\mathrm{CH}_{3}-4\right.$ '), $23.04\left(\mathrm{CH}_{3}-2\right), 113.68(\mathrm{C}-4 \mathrm{a}), 118.72(\mathrm{CH}-5), 124.95$ (CH-6), 125.45 (CH-2' and $\mathrm{CH}-6$ '), 130.49 (CH-3' and $\mathrm{CH}^{\prime}-5$ '), $130.82(\mathrm{CH}-8), 135.55\left(\mathrm{C}_{\text {quat }}\right), 137.06(\mathrm{CH}-7)$, $138.06\left(C_{\text {quat }}\right), 141.71(C-8 \mathrm{a}), 161.01(C-2), 163.63(C-4)$; Anal. calc. C 77.08, H 6.06, for $\mathrm{C}_{16} \mathrm{H}_{15} \mathrm{~N}_{3}$, found C 77.19, H 6.12.

2-Methyl- $\mathrm{N}$-m-tolylquinazolin-4-amine (4). m. p. $239-242{ }^{\circ} \mathrm{C} ; \mathrm{R}_{\mathrm{f}} 0.41$ (ethylacetate/hexane 1:1), $\mathrm{R}_{\mathrm{f}} 0.26$ (ethylacetate/hexane 1:2); ${ }^{1} \mathrm{H}$ NMR 2.406 (s, 3H, $\mathrm{CH}_{3}-3$ '), 2.677 (s, 3H, $\mathrm{CH}_{3}-2$ ), 7.169 (d, 1H, J 7.5, CH-6'), 7.355 (dd, 1H, J 8.4, 7.5, CH-5'), 7.531 (s, 1H, CH-2'), 7.548 (d, 1H, J 8.4, CH-4'), 7.804 (m, 2H, CH-5 and $\mathrm{CH}-7$ ), 8.056 (ddd, 1H, J 8.3, 7.5, 1.1, CH-6), 8.584 (dd, 1H, J 9.2, 1.4, CH-8); ${ }^{13} \mathrm{C}$ NMR $21.49\left(\mathrm{CH}_{3}-3\right.$ '), $22.68\left(\mathrm{CH}_{3}-2\right), 113.54(\mathrm{C}-4 \mathrm{a}), 120.03(\mathrm{CH}-$ 
5), 122.93 (CH-6'), 125.19 (CH-6), 126.23 (CH-2'), 128.96 (CH-4'), 129.73 (CH-5'), 129.86 $(\mathrm{CH}-8), 137.47$ (CH-7), $137.74\left(C_{\text {quat }}\right), 140.22$ (C-8a), $140.55\left(C_{\text {quat }}\right), 161.28(C-2), 163.45(C-$ 4); Anal. calc. C 77.08, H 6.06, for $\mathrm{C}_{16} \mathrm{H}_{15} \mathrm{~N}_{3}$, found $\mathrm{C} 77.14, \mathrm{H} 6.09$.

2-Methyl- $\mathrm{N}$-o-tolylquinazolin-4-amine (5). m. p. $186-190{ }^{\circ} \mathrm{C} ; \mathrm{R}_{\mathrm{f}} 0.54$ (ethylacetate/hexane 1:1), $\mathrm{R}_{\mathrm{f}} 0.37$ (ethylacetate/hexane 1:2); ${ }^{1} \mathrm{H}$ NMR 2.293 (s, 3H, $\mathrm{CH}_{3}-2$ '), 2.526 (s, 3H, $\mathrm{CH}_{3}-2$ ), 6.794 (dd, 1H, J 8.0, 1.6, CH), 7.172 (m, 1H, CH), 7.302 (m, 1H, CH), 7.445 (dd, 1H, J 8.2, 1.7, $\mathrm{CH}), 7.733(\mathrm{~d}+\mathrm{t}, 2 \mathrm{H}, \mathrm{CH}-5$ and $\mathrm{CH}-7), 7.982(\mathrm{ddd}, 1 \mathrm{H}, \mathrm{J} 8.2,7.8,1.2, \mathrm{CH}-6), 8.456(\mathrm{dd}, 1 \mathrm{H}, \mathrm{J}$ 8.2, 1.3, $\mathrm{CH}-8) ;{ }^{13} \mathrm{C}$ NMR $17.72\left(\mathrm{CH}_{3}-2\right.$ '), $23.27\left(\mathrm{CH}_{3}-2\right), 113.42$ (C-4a), 124.67 (CH-5), 125.37 $(\mathrm{CH}-6), 126.36(\mathrm{CH}), 128.55(\mathrm{CH}), 129.11(\mathrm{CH}), 129.48(\mathrm{CH}), 129.76(\mathrm{CH}-8), 134.60\left(C_{\text {quat }}\right)$, $136.42\left(C_{\text {quat }}\right), 136.81(C \mathrm{H}-7), 142.69(C-8 \mathrm{a}), 161.96(C-2), 163.96(C-4)$; Anal. calc. C 77.08, H 6.06 , for $\mathrm{C}_{16} \mathrm{H}_{15} \mathrm{~N}_{3}$, found $\mathrm{C} 77.02, \mathrm{H} 6.21$.

$\mathrm{N}$-(2,6-Dimethylphenyl)-2-methylquinazolin-4-amine (6). m. $\quad$ p. $260-263 \quad{ }^{\circ} \mathrm{C} ; \mathrm{R}_{\mathrm{f}} \quad 0.39$ (ethylacetate/hexane 1:1); ${ }^{1} \mathrm{H}$ NMR 2.233 (s, 6H, $\mathrm{CH}_{3}-2$ ' and $\mathrm{CH}_{3}-6$ '), 2.574 (s, 3H, $\mathrm{CH}_{3}-2$ ), 7.218 (m, 3H, CH-3', $\mathrm{CH}-4^{\prime}$ ' and $\mathrm{CH}-5^{\prime}$ '), 7.850 (m, 2H, CH-5 and $\mathrm{CH}-7$ ), 8.105 (ddd, 1H, J 8.3, 7.3, 1.3, $\mathrm{CH}-6), 8.585(\mathrm{dd}, 1 \mathrm{H}, \mathrm{J} 7.8,1.4, \mathrm{CH}-8) ;{ }^{13} \mathrm{C} \mathrm{NMR} 18.30\left(\mathrm{CH}_{3}-2^{\prime}\right.$ and $\mathrm{CH}_{3}-6$ '), 22.61 $\left(\mathrm{CH}_{3}-2\right), 112.88(\mathrm{C}-4 \mathrm{a}), 120.08(\mathrm{CH}-5), 125.17(\mathrm{CH}-6), 129.50\left(\mathrm{CH}_{-3}{ }^{\prime}\right.$ and $\left.\mathrm{CH}-5^{\prime}\right), 129.60(\mathrm{CH}-$ 8), $129.90\left(\mathrm{CH}^{-4}\right), 135.26\left(C_{\text {quat }}\right), 136.83\left(C-2^{\prime}\right.$ and $C-6$ '), $137.72(C \mathrm{H}-7), 140.43(C-8 \mathrm{a})$, 162.36 (C-2), 163.88 (C-4); Anal. calc. C 77.54, $\mathrm{H}$ 6.51, for $\mathrm{C}_{17} \mathrm{H}_{17} \mathrm{~N}_{3}$, found $\mathrm{C} 77.61, \mathrm{H}$ 6.42.

$\mathrm{N}$-(4-Methoxyphenyl)-2-methylquinazolin-4-amine (7). $\quad$ m. p. $\quad 219-220{ }^{\circ} \mathrm{C} ; \quad \mathrm{R}_{\mathrm{f}} \quad 0.43$ (ethylacetate/hexane 1:1), $\mathrm{R}_{\mathrm{f}} 0.30$ (ethylacetate/hexane 1:2); ${ }^{1} \mathrm{H}$ NMR 2.657 (s, 3H, $\mathrm{CH}_{3}-2$ ), 3.848 (s, 3H, $\mathrm{OCH}_{3}-4^{\prime}$ ), 7.023 (d, 2H, J 9.1, $\mathrm{CH}-3$ ' and $\mathrm{CH}-5$ '), 7.638 (d, 2H, J 9.1, $\mathrm{CH}-2^{\prime}$ ' and CH-6'), 7.781 (d+t, 2H, CH-5 и CH-7), 8.035 (t, 1H, J 8.3, CH-6), 8.538 (d, 1H, J 8.3, CH-8); ${ }^{13} \mathrm{C}$ NMR $22.77\left(\mathrm{CH}_{3}-2\right), 56.05\left(\mathrm{OCH}_{3}-4{ }^{\prime}\right), 113.56(\mathrm{C}-4 \mathrm{a}), 115.14\left(\mathrm{CH}-3\right.$ ' and $\left.\mathrm{CH}-5{ }^{\prime}\right), 116.12$ $\left(C_{\text {quat }}\right), 120.21(\mathrm{CH}-5), 125.04(\mathrm{CH}-6), 127.13\left(\mathrm{CH}-2\right.$ ' and $\left.\mathrm{CH}-6{ }^{\prime}\right), 129.56(\mathrm{CH}-8), 130.65$ $\left(C_{\text {quat }}\right), 137.25$ (CH-7), 140.81 (C-8a), 160.05 (C-2), 163.46 (C-4); Anal. calc. C 72.43, H 5.70, for $\mathrm{C}_{16} \mathrm{H}_{15} \mathrm{~N}_{3} \mathrm{O}$, found $\mathrm{C} 72.51, \mathrm{H} 5.62$.

$\mathrm{N}$-(3-Methoxyphenyl)-2-methylquinazolin-4-amine (8). $\quad$ m. $\quad$ p. $220-221 \quad{ }^{\circ} \mathrm{C} ; \quad \mathrm{R}_{\mathrm{f}} \quad 0.35$ (ethylacetate/hexane 1:1), $\mathrm{R}_{\mathrm{f}} 0.20$ (ethylacetate/hexane 1:2); ${ }^{1} \mathrm{H}$ NMR 2.703 (s, 3H, $\mathrm{CH}_{3}-2$ ), 3.848 (s, 3H, $\mathrm{OCH}_{3}-3^{\prime}$ '), 6.292 (dd, $1 \mathrm{H}, \mathrm{CH}-6$ ') 7.388 (m, 3H, CH-2', $\mathrm{CH}-4^{\prime}$ ' and $\mathrm{CH}-\mathrm{C}^{\prime}$ '), 7.816 $(\mathrm{d}+\mathrm{t}, 2 \mathrm{H}, \mathrm{CH}-5$ and $\mathrm{CH}-7), 8.068$ (t, $1 \mathrm{H}, \mathrm{J} 7.5, \mathrm{CH}-6), 8.600(\mathrm{~d}, 1 \mathrm{H}, \mathrm{J} 8.2, \mathrm{CH}-8) ;{ }^{13} \mathrm{C}$ NMR $22.68\left(\mathrm{CH}_{3}-2\right), 56.02\left(\mathrm{OCH}_{3}-3\right), 111.71(\mathrm{CH}), 113.58(\mathrm{C}-4 \mathrm{a}), 113.73(\mathrm{CH}), 117.84(\mathrm{CH})$, $120.00(\mathrm{CH}-5), 125.19(\mathrm{CH}-6), 129.82(\mathrm{CH}-8), 130.79(\mathrm{CH}), 137.56(\mathrm{CH}-7), 138.91\left(C_{\text {quat }}\right)$, 140.48 (C-8a), 161.29 (C-3'), 161.61 (C-2), 163.48 (C-4); Anal. calc. C 72.43, H 5.70, for $\mathrm{C}_{16} \mathrm{H}_{15} \mathrm{~N}_{3} \mathrm{O}$, found $\mathrm{C} 72.32$, $\mathrm{H} 5.81$.

$\mathrm{N}$-(2-Methoxyphenyl)-2-methylquinazolin-4-amine (9). $\quad$ m. $\quad$ p. $\quad 218-219 \quad{ }^{\circ} \mathrm{C} ; \quad \mathrm{R}_{\mathrm{f}} \quad 0.27$ (ethylacetate/hexane 1:1); ${ }^{1} \mathrm{H}$ NMR 2.412 (s, $3 \mathrm{H}, \mathrm{CH}_{3}-2$ ), 3.672 (s, $3 \mathrm{H}, \mathrm{OCH}_{3}-2$ '), 7.330 (m, $2 \mathrm{H}$, $\mathrm{CH}), 7.477(\mathrm{~m}, 2 \mathrm{H}, \mathrm{CH}), 7.600(\mathrm{~d}+\mathrm{t}, 2 \mathrm{H}, \mathrm{CH}-5$ and $\mathrm{CH}-7), 7.859$ (ddd, $1 \mathrm{H}, \mathrm{J} 8.1,7.4,1.0, \mathrm{CH}-$ 6), $8.302(\mathrm{dd}, 1 \mathrm{H}, \mathrm{J} 7.8,1.1, \mathrm{CH}-8) ;{ }^{13} \mathrm{C}$ NMR $22.64\left(\mathrm{CH}_{3}-2\right), 56.48\left(\mathrm{OCH}_{3}-2\right), 113.04(\mathrm{CH})$, $113.31(\mathrm{C}-4 \mathrm{a}), 120.09(\mathrm{CH}-5), 121.65(\mathrm{CH}), 125.09(\mathrm{CH}-6), 126.17\left(C_{\text {quat }}\right), 128.38(\mathrm{CH}), 129.70$ 
(CH-8), $130.23(\mathrm{CH}), 137.49$ (CH-7), 140.58 (C-8a), 155.34 ( $\left.C_{\text {quat }}\right), 161.61(C-2), 163.44(C-4)$; Anal. calc. C 72.43, $\mathrm{H} 5.70$, for $\mathrm{C}_{16} \mathrm{H}_{15} \mathrm{~N}_{3} \mathrm{O}$, found $\mathrm{C} 72.48, \mathrm{H}$ 5.65.

2-Methyl- $N$-(3,4,5-trimethoxyphenyl)quinazolin-4-amine (10). m. p. $256-260{ }^{\circ} \mathrm{C} ; \mathrm{R}_{\mathrm{f}} 0.29$ (ethylacetate/hexane 1:1); ${ }^{1} \mathrm{H}$ NMR 2.720 (s, 3H, $\left.\mathrm{CH}_{3}-2\right), 3.804$ (s, 3H, OCH$H_{3} 4^{\prime}$ ), 3.881 (s, 6H, $\left(\mathrm{OCH}_{3}\right)_{2}-3^{\prime}, 5^{\prime}$ '), 7.203 (s, 2H, CH-2' and $\mathrm{CH}-6$ '), 7.794 (d, 1H, J 7.9, CH-5), 7.810 (t, 1H, J 8.1, $\mathrm{CH}-7), 8.060$ (t, 1H, J 7.9, $\mathrm{CH}-6), 8.609$ (d, 1H, J 8.1, $\mathrm{CH}-8) ;{ }^{13} \mathrm{C}$ NMR $22.87\left(\mathrm{CH}_{3}\right), 56.85$ $\left(\left(\mathrm{OCH}_{3}\right)_{2}-3^{\prime}, 5^{\prime}\right), 61.28\left(\mathrm{OCH}_{3}-4\right.$ '), $103.42(\mathrm{CH}-2$ ' and $\mathrm{CH}-6$ '), $113.66(C-4 \mathrm{a}), 120.26(\mathrm{CH}-5)$, 125.12 (CH-6), $129.71(C \mathrm{H}-8), 134.00\left(C_{\text {quat }}\right), 137.40(C \mathrm{H}-7), 137.94\left(C_{\text {quat }}\right), 140.80(C-8 \mathrm{a})$, $154.56\left(C_{\text {quat }}\right), 161.00(C-2), 163.47(C-4)$; Anal. calc. C 66.45, H 5.89, for $\mathrm{C}_{18} \mathrm{H}_{19} \mathrm{~N}_{3} \mathrm{O}_{3}$, found C 66.27, H 5.96.

2-Methyl- $\boldsymbol{N}$-(4-nitrophenyl)quinazolin-4-amine $\quad$ (11):.m. $\quad$ p. $\quad 138-141 \quad{ }^{\circ} \mathrm{C} ; \quad \mathrm{R}_{\mathrm{f}} \quad 0.39$ (ethylacetate/hexane 1:1); ${ }^{1} \mathrm{H}$ NMR 2.764 (s, 3H, $\left.\mathrm{CH}_{3}-2\right), 7.854$ (m, 2H, CH-3' and $\mathrm{CH}-5$ '), $8.122(\mathrm{~m}, 3 \mathrm{H}, \mathrm{CH}-5, \mathrm{CH}-7$ and $\mathrm{CH}), 8.358(\mathrm{~m}, 2 \mathrm{H}, \mathrm{CH}-6$ and $\mathrm{CH}), 8.624(\mathrm{dd}, 1 \mathrm{H}, \mathrm{J}$ 8.9, 1.2, $\mathrm{CH}-8) ;{ }^{13} \mathrm{C}$ NMR $23.20\left(\mathrm{CH}_{3}-2\right), 113.66(\mathrm{C}-4 \mathrm{a}), 121.32(\mathrm{CH}-5), 124.99(\mathrm{CH}-6), 125.21(2 \mathrm{CH})$, $125.45(2 \mathrm{CH}), 129.85(\mathrm{CH}-8), 137.62(\mathrm{CH}-7), 144.45(C-8 \mathrm{a}), 148.68\left(C_{\text {quat }}\right), 156.85\left(C_{\text {quat }}\right)$, 161.26 (C-2), 164.05 (C-4); Anal. calc. C 64.28, $\mathrm{H} 4.32$, for $\mathrm{C}_{15} \mathrm{H}_{12} \mathrm{~N}_{4} \mathrm{O}_{2}$, found $\mathrm{C}$ 64.16, $\mathrm{H} 4.44$. 2-Methyl- $N$-(2-nitrophenyl)quinazolin-4-amine $\quad$ (12). $\quad$ m. $\quad$ p. $\quad 145-148 \quad{ }^{\circ} \mathrm{C} ; \quad \mathrm{R}_{\mathrm{f}} \quad 0.57$ (ethylacetate/hexane 1:1); ${ }^{1} \mathrm{H}$ NMR 2.610 (s, 3H, $\mathrm{CH}_{3}-2$ ), 7.652 (ddd, 1H, J 7.8, 6.4, 2.0, CH), $7.862(\mathrm{~m}, 4 \mathrm{H}, \mathrm{CH}-5, \mathrm{CH}-7$ and $2 \mathrm{CH}), 8.138(\mathrm{t}, 1 \mathrm{H}, \mathrm{J} 7.1, \mathrm{CH}-6), 8.180(\mathrm{~d}, 1 \mathrm{H}, \mathrm{J}$ 8.1, CH), 8.584 (d, 1H, J 8.3, CH-8); ${ }^{13} \mathrm{C}$ NMR $22.44\left(\mathrm{CH}_{3}-2\right), 113.28(\mathrm{C}-4 \mathrm{a}), 120.11(\mathrm{CH}-5), 120.25(\mathrm{CH})$, $125.22(\mathrm{CH}-6), 126.51(\mathrm{CH}), 129.79(\mathrm{CH}-8), 130.31(\mathrm{CH}), 135.39(\mathrm{CH}), 136.63\left(C_{\text {quat }}\right), 138.21$ (CH-7), 140.68 (C-8a), 146.49 ( $\left.C_{\text {quat }}\right), 161.85$ (C-2), 163.62 (C-4); Anal. calc. C 64.28, H 4.32, for $\mathrm{C}_{15} \mathrm{H}_{12} \mathrm{~N}_{4} \mathrm{O}_{2}$, found $\mathrm{C} 64.22, \mathrm{H} 4.39$.

$\mathrm{N}$-(4-Fluorophenyl)-2-methylquinazolin-4-amine $\quad$ (13). $\quad$ m. $\quad$ p. $184-186 \quad{ }^{\circ} \mathrm{C} ; \quad \mathrm{R}_{\mathrm{f}} \quad 0.37$ (ethylacetate/hexane 1:1); ${ }^{1} \mathrm{H}$ NMR 2.677 (s, 3H, $\mathrm{CH}_{3}-2$ ), 7.208 (d, 2H, J 8.9, CH-2' or CH-6'), 7.243 (d, 2H, J 8.6, CH-2' or CH-6'), 7.765 (m, 4H, $\mathrm{CH}-5, \mathrm{CH}-7, \mathrm{CH}-3$ ' and $\mathrm{CH}-5$ '), 8.061 (ddd, $1 \mathrm{H}, \mathrm{J} 8.1,7.4,1.2, \mathrm{CH}-6), 8.612$ (dd, $1 \mathrm{H}, \mathrm{J} 8.4,1.3, \mathrm{CH}-8) ;{ }^{13} \mathrm{C}$ NMR $22.78\left(\mathrm{CH}_{3}-2\right), 113.51(\mathrm{C}$ -

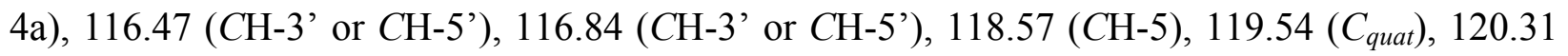
(CH-6), 125.11 (CH-8), 127.85 (CH-2' or $\mathrm{CH}-6$ '), 127.98 (CH-2' or $\mathrm{CH}-6$ '), 134.08 ( $\left.C_{\text {quat }}\right)$, $137.45(\mathrm{CH}-7), 140.90(C-8 \mathrm{a}), 161.35(C-2), 163.60(C-4)$; Anal. calc. C 71.13, H 4.78 for $\mathrm{C}_{15} \mathrm{H}_{12} \mathrm{FN}_{3}$, found $\mathrm{C} 71.03, \mathrm{H} 4.84$.

$\boldsymbol{N}$-(4-Bromophenyl)-2-methylquinazolin-4-amine (14). $\quad$ m. $\quad$ p. $273-275 \quad{ }^{\circ} \mathrm{C} ; \quad \mathrm{R}_{\mathrm{f}} \quad 0.44$ (ethylacetate/hexane 1:1); ${ }^{1} \mathrm{H}$ NMR 2.702 (s, 3H, $\left.\mathrm{CH}_{3}-2\right), 7.616$ (d, 2H, J 8.9, CH-2' and CH-6'), 7.723 (d, 2H, J 8.9, $\mathrm{CH}-3^{\prime}$ ' and $\left.\mathrm{CH}-5^{\prime}\right), 7.822$ (m, 2H, $\mathrm{CH}-5$ and $\left.\mathrm{CH}-7\right), 8.071$ (ddd, 1H, J 8.3, 7.3, 1.2, $\mathrm{CH}-6), 8.612(\mathrm{dd}, 1 \mathrm{H}, \mathrm{J} 8.8,1.3, \mathrm{CH}-8) ;{ }^{13} \mathrm{C} \mathrm{NMR} 22.80\left(\mathrm{CH}_{3}-2\right), 113.62(\mathrm{C}-4 \mathrm{a})$, 118.37 (CH-5), 120.33 (CH-6), 121.08 ( $\left.C_{\text {quat }}\right), 125.16(\mathrm{CH}-8), 127.46\left(\mathrm{CH}_{-}\right.$' ' and $\mathrm{CH}-6$ '), 133.05 (CH-3' and $C H-5 '), 137.24\left(C_{\text {quat }}\right), 137.57$ (CH-7), 140.92 (C-8a), $161.23(C-2), 163.60$ (C-4); Anal. calc. C 57.34, $\mathrm{H} 3.85$, for $\mathrm{C}_{15} \mathrm{H}_{12} \mathrm{BrN}_{3}$, found C 57.22, H 3.99.

$\boldsymbol{N}$-(4-Chlorophenyl)-2-methylquinazolin-4-amine (15). $\quad$ m. $\quad$ p. $\quad 203-205 \quad{ }^{\circ} \mathrm{C} ; \quad \mathrm{R}_{\mathrm{f}} \quad 0.43$ (ethylacetate/hexane 1:1); ${ }^{1} \mathrm{H}$ NMR 2.704 (s, 3H, $\mathrm{CH}_{3}-2$ ), 7.470 (d, 2H, J 8.9, CH-2' and CH-6'), 
7.778 (d, 2H, J 8.9, $\mathrm{CH}-3$ ' and $\mathrm{CH}-5$ '), 7.820 (m, 2H, CH-5 and $\mathrm{CH}-7$ ), 8.070 (t, 1H, J 7.8, $\mathrm{CH}-$ 6), 8.612 (d, 1H, J 8.3, CH-8); ${ }^{13} \mathrm{C}$ NMR $22.70\left(\mathrm{CH}_{3}-2\right), 113.54$ (C-4a), 118.75 (CH-5), 120.09 (CH-6), 125.21 (CH-8), 127.25 (CH-2' and $\left.\mathrm{CH}^{\prime} 6^{\prime}\right), 130.00$ (CH-3' and $\left.\mathrm{CH}-\mathrm{C}^{\prime}\right), 133.38\left(\mathrm{C}_{\text {quat }}\right.$ ), $136.66\left(C_{\text {quat }}\right), 137.62(\mathrm{CH}-7), 140.56$ (C-8a), $161.26(C-2), 163.51(C-4)$; Anal. calc. C 66.79, H 4.48, for $\mathrm{C}_{15} \mathrm{H}_{12} \mathrm{ClN}_{3}$, found $\mathrm{C} 66.63, \mathrm{H} 4.56$.

$\mathrm{N}$-(2,3-Dichlorophenyl)-2-methylquinazolin-4-amine (16). $\quad$ m. p. $\quad 267-268{ }^{\circ} \mathrm{C} ; \mathrm{R}_{\mathrm{f}} \quad 0.53$ (ethylacetate/hexane 1:1); ${ }^{1} \mathrm{H}$ NMR 2.619 (s, 3H, $\left.\mathrm{CH}_{3}-2\right), 7.476$ (d, $1 \mathrm{H}, \mathrm{J}$ 8.0, $\mathrm{CH}$ ), 7.626 (m, 2H, J 8.9, CH), 7.856 (m, 2H, CH-5 and $\mathrm{CH}-7$ ), 8.108 (dt, 1H, J 8.2, 1.3, CH-6), 8.553 (dd, 1H, J 8.8, 1.3, $\mathrm{CH}-8) ;{ }^{13} \mathrm{C}$ NMR $22.81\left(\mathrm{CH}_{3}-2\right), 113.18(\mathrm{C}-4 \mathrm{a}), 120.61(\mathrm{CH}-5), 125.13(\mathrm{CH}-6), 125.26$ $\left(C_{\text {quat }}\right), 129.05(\mathrm{CH}-8), 129.26(\mathrm{CH}), 129.97(\mathrm{CH}), 131.23(\mathrm{CH}), 134.78\left(C_{\text {quat }}\right), 137.19\left(C_{\text {quat }}\right)$, 137.87 (CH-7), 141.30 (C-8a), 161.42 (C-2), 163.88 (C-4); Anal. calc. C 59.23, H 3.65, for $\mathrm{C}_{15} \mathrm{H}_{11} \mathrm{Cl}_{2} \mathrm{~N}_{3}$, found $\mathrm{C} 59.18, \mathrm{H} 3.72$.

$\mathrm{N}$-(2,6-Dichlorophenyl)-2-methylquinazolin-4-amine (17). m. p. $\quad 218-220{ }^{\circ} \mathrm{C} ; \mathrm{R}_{\mathrm{f}} \quad 0.45$ (ethylacetate/hexane 1:1); ${ }^{1} \mathrm{H}$ NMR 2.703 (s, 3H, $\left.\mathrm{CH}_{3}-2\right)$, 7.486 (dd, $2 \mathrm{H}, \mathrm{J}$ 6.9, 1.8, $\mathrm{CH}-3$ ' ' and CH-5'), 7.791 (m, 3H, CH-5, CH-7 and $\mathrm{CH}-4$ '), 8.077 (t, 1H, J 6.7, CH-6), 8.588 (d, 1H, J 8.5, $\mathrm{CH}-8) ;{ }^{13} \mathrm{C}$ NMR $22.74\left(\mathrm{CH}_{3}-2\right), 113.61(\mathrm{C}-4 \mathrm{a}), 118.61(\mathrm{CH}-5), 120.24(\mathrm{CH}-6), 125.14(\mathrm{CH}-8)$, 129.84 (CH-4'), $130.05\left(\mathrm{CH}_{-}{ }^{\prime}\right.$ ' and $\mathrm{CH}-5$ '), $133.43\left(C_{\text {quat }}\right), 136.72\left(C_{\text {quat }}\right), 137.61(\mathrm{CH}-7)$, 140.82 (C-8a), $161.31(C-2), 163.62(C-4)$; Anal. calc. C 59.23, $\mathrm{H} 3.65$, for $\mathrm{C}_{15} \mathrm{H}_{11} \mathrm{Cl}_{2} \mathrm{~N}_{3}$, found C 59.13, H 3.69.

2-Methyl- $\boldsymbol{N}$-(naphthalen-1-yl)quinazolin-4-amine (19). $\quad$ m. p. $\quad 222-224 \quad{ }^{\circ} \mathrm{C} ; \quad \mathrm{R}_{\mathrm{f}} \quad 0.32$ (ethylacetate/hexane 1:1); ${ }^{1} \mathrm{H}$ NMR 2.449 (s, 3H, $\left.\mathrm{CH}_{3}-2\right), 7.561(\mathrm{~m}, 4 \mathrm{H}, 4 \mathrm{CH}), 7.971(\mathrm{~m}, 6 \mathrm{H}$, $\mathrm{CH}-5, \mathrm{CH}-6, \mathrm{CH}-7$ and $3 \mathrm{CH}), 8.657(\mathrm{~d}, 1 \mathrm{H}, \mathrm{J} 8,2, \mathrm{CH}-8) ;{ }^{13} \mathrm{C}$ NMR $22.63\left(\mathrm{CH}_{3}-2\right), 113.31(\mathrm{C}-$ 4a), $120.34(\mathrm{CH}), 123.68(\mathrm{CH}-5), 125.28(\mathrm{CH}-6), 126.18(\mathrm{CH}), 126.62(\mathrm{CH}), 127.67(\mathrm{CH})$, $128.00(\mathrm{CH}), 129.26\left(C_{\text {quat }}\right), 129.61(\mathrm{CH}-8), 129.75(\mathrm{CH}), 129.86(\mathrm{CH}), 131.03\left(C_{\text {quat }}\right), 135.94$ $\left(C_{\text {quat }}\right), 137.56(C \mathrm{H}-7), 141.01$ (C-8a), 163.28 (C-2), 163.68 (C-4); Anal. calc. C 79.98, H 5.30, for $\mathrm{C}_{19} \mathrm{H}_{15} \mathrm{~N}_{3}$, found $\mathrm{C} 79.92, \mathrm{H} 5.39$.

2-Methyl- $\mathrm{N}$-(naphthalen-2-yl)quinazolin-4-amine (20). $\quad$ m. $\quad$ P. $223-226 \quad{ }^{\circ} \mathrm{C} ; \quad \mathrm{R}_{\mathrm{f}} \quad 0.39$ (ethylacetate/hexane 1:1); ${ }^{1} \mathrm{H}$ NMR 2.667 (s, 3H, $\left.\mathrm{CH}_{3}-2\right), 7.517$ (m, 2H, CH), 7.924 (m, 7H, CH5, $\mathrm{CH}-6, \mathrm{CH}-7$ and $4 \mathrm{CH}$ ), 8.257 (d, $1 \mathrm{H}, \mathrm{J} 1,9, \mathrm{CH}-1$ '), 8.581 (d, $1 \mathrm{H}, \mathrm{J} 8,3, \mathrm{CH}-8) ;{ }^{13} \mathrm{C}$ NMR $23.15\left(\mathrm{CH}_{3}-2\right), 113.80(\mathrm{C}-4 \mathrm{a}), 121.07(\mathrm{CH}), 123.20(\mathrm{CH}-5), 124.25(\mathrm{CH}), 125.00(\mathrm{CH}-6), 127.11$ $\left(C_{\text {quat }}\right), 127.38(\mathrm{CH}), 127.82(\mathrm{CH}), 128.78(\mathrm{CH}), 128.98(\mathrm{CH}), 129.45(\mathrm{CH}), 129.62(\mathrm{CH}-8)$, 129.90 ( $\left.C_{\text {quat }}\right), 134.89\left(C_{\text {quat }}\right), 137.11(C \mathrm{H}-7), 142.02$ (C-8a), $161.10(C-2), 163.74$ (C-4); Anal. calc. C 79.98, H 5.30, for $\mathrm{C}_{19} \mathrm{H}_{15} \mathrm{~N}_{3}$, found C 80.11, H 5.23.

$\mathrm{N}$-Benzyl-2-methylquinazolin-4-amine (21). ${ }^{33} \mathrm{~m}$. P. $168-170{ }^{\circ} \mathrm{C}$ (lit. ${ }^{33} 186-187{ }^{\circ} \mathrm{C}$ ); $\mathrm{R}_{\mathrm{f}} 0.50$ (ethylacetate); ${ }^{1} \mathrm{H}$ NMR 2.520 (s, 3H, $\left.\mathrm{CH}_{3}-2\right), 4.856\left(\mathrm{~s}, 2 \mathrm{H}, \mathrm{CH}_{2}\right), 7.331(\mathrm{~m}, 6 \mathrm{H}, \mathrm{CH}-5$ and 5 $\mathrm{CH}), 7.684$ (m, 2H, CH-6 and $\mathrm{CH}-7), 8.082$ (dd, $1 \mathrm{H}, \mathrm{J}$ 8.3, 1.3, $\mathrm{CH}-8) ;{ }^{13} \mathrm{C}$ NMR $25.94\left(\mathrm{CH}_{3}-2\right)$, $45.34\left(\mathrm{CH}_{2}\right), 114.48(\mathrm{C}-4 \mathrm{a}), 123.30(\mathrm{CH}-5), 126.54(\mathrm{CH}-6), 126.91\left(\mathrm{CH}_{-} \mathbf{C}^{\prime}\right), 128.11(\mathrm{CH}-8)$, $128.75(2 \mathrm{CH}), 129.47(2 \mathrm{CH}), 134.02(\mathrm{CH}-7), 140.55$ (C-8a), 150.37 ( $\left.C_{\text {quat }}\right), 161.49(\mathrm{C}-2)$, 165.62 (C-4); Anal. calc. C 77.08, H 6.06, for $\mathrm{C}_{16} \mathrm{H}_{15} \mathrm{~N}_{3}$, found C 77.01, H 6.18. 
$\mathbf{N}$-(3,4-Dimethoxyphenethyl)-2-methylquinazolin-4-amine (22). m. p. $132-135{ }^{\circ} \mathrm{C} ; \mathrm{R}_{\mathrm{f}} 0.30$ (ethylacetate); ${ }^{1} \mathrm{H}$ NMR 2.534 (s, 3H, $\left.\mathrm{CH}_{3}-2\right), 2.936$ (t, 2H, J 7.6, $\mathrm{CH}_{2}$ ), 3.723 (s, 3H OCH$)$, 3.768 (s, 3H OCH 3$), 3.824$ (q, 2H, J 7.6, 8.6, $\mathrm{CH}_{2}$ ), 6.818 (m, 3H, CH-2', $\mathrm{CH}-5$ ' и С $\mathrm{C}-6$ ') 7.404 (ddd, 1H, J 8.2, 6.8, 1.4, CH-7), $7.672(\mathrm{~m}, 2 \mathrm{H}, \mathrm{CH}-5$ and $\mathrm{CH}-6), 7.975$ (dd, 1H, J 8.3, 0.9, CH8); ${ }^{13} \mathrm{C}$ NMR $26.00\left(\mathrm{CH}_{3}-2\right), 35.79\left(\mathrm{CH}_{2}\right), 43.77\left(\mathrm{CH}_{2}\right), 56.36\left(\mathrm{OCH}_{3}\right), 56.59\left(\mathrm{OCH}_{3}\right), 113.26$ $(\mathrm{CH}), 114.04(\mathrm{CH}), 114.52(\mathrm{C}-4 \mathrm{a}), 122.33(\mathrm{CH}), 123.14(\mathrm{CH}-5), 126.41(\mathrm{CH}-6), 126.93(\mathrm{CH}-8)$, 133.91 (CH-7), 149.06 (C-8a), 150.29 ( $\left.C_{\text {quat }}\right), 150.40\left(C_{\text {quat }}\right), 161.44(C-2), 165.56(C-4)$; Anal. calc. C 70.57, $\mathrm{H} 6.55$, for $\mathrm{C}_{19} \mathrm{H}_{21} \mathrm{~N}_{3} \mathrm{O}$, found $\mathrm{C} 70.42, \mathrm{H} 6.66$.

\section{References and Footnotes}

1. Jhone, S. In Progress in Drug Research, Jucker, E., Ed.; Birkauser Verlag: Basel, 1982, Vol. 26, 259-341.

2. Brown, D. J. The Chemistry of Heterocyclic Compounds, J. Wiley \& Sons: New York, 1996.

3. Armarego, W. Fused Pyrimidines: Part I - Quinazolines, Interscience Publishers: New York - London - Sydney, 1967.

4. Nakagami, K.; Yokoi, S.; Nishimura, K.; Nagai, S.; Honda, T.; Oda, K.; Kobayashi, R.; Kojima, M. US Patent 4323680, 1982.

5. Haley, G. J. US Patent 5373011, 1994.

6. Palanki, M.; Suto, M. US Patent 5939421, 1999.

7. Myers, M. R.; Spada, A.; Maguire, M; Persons, P.; Zilberstein, A.; Hsu, C.-Y.-J.; Johnson, S. E. US Patent 5714493, 1998. .

8. Baker, A. J. US Patent 5932574, 1999.

9. Baker, A. J. US Patent 5942514, 1999.

10. Nauta, W. T. US Patent 3980650, 1976.

11. Mizogami, S.; Hiranuma, H.; Sekiya, T.; Hanazuka, M. US Patent 4607034, 1986.

12. Berger, M.; Albrecht, B.; Breces, A.; Neruda, W.; Woisetschläger, M. J. Med. Chem. 2001, 44, 3031.

13. Bridges, A. J. Chem. Rev. 2001, 101, 2541.

14. Comprehensive Heterocyclic Chemistry II, Katritzky, A. R.; Rees, C. W., Eds; Pergamon Press: Oxford, 1996.

15. Katritzky, A. R.; Pozharski, A. F. Handbook of Heterocyclic Chemistry, $2^{\text {nd }}$ Edn., Pergamon: New York, 2000.

16. Caddick, S. Tetrahedron 1995, 51, 10403.

17. Deshayes, S.; Liagre, M.; Loupy, A.; Luche, J.-L.; Petit, A. Tetrahedron 1999, 55, 10851.

18. Lidstrom, P.; Tierney, J.; Wathey, B.; Westman, J. Tetrahedron 2001, 57, 9225.

19. Kirschning, A.; Monenschein, H.; Wittenberg, R. Angew. Chem., Int. Ed. 2001, 40, 650.

20. Kappe, C. O. Angew. Chem. Int. Ed. 2004, 43, 6250. 
21. Kappe, C. O.; Stadler, A. Microwaves in Organic and Medicinal Chemistry, Methods and Principles in Medicinal Chemistry, Vol. 25, Mannhold, R.; Kubinyi, H.; Folkers, G.,Eds.; 1st ed., Wiley-VCH: Weinheim, Germany, 2005.

22. Loupy, A.; Petit, A.; Hamelin, J.; Texier-Boullet, F.; Jacquault, P.; Mathe, D. Synthesis 1998, 1213.

23. Varma, R. S. Green Chemistry 1999, 1, 43.

24. Tanaka, K.; Toda, F. Chem. Rev. 2000, 100, 1025.

25. Jolivet-Fouchet, S.; Hamelin, J.; Texier-Boullet, F.; Toupet, L.; Jacquault, P. Tetrahedron 1998, $54,4561$.

26. Varma, R. J. Heterocyclic Chem. 1999, 36, 1565.

27. Diaz-Ortiz, A.; de la Hoz, A.; Langa, F. Green Chem. 2000, 2, 165.

28. Katritzky, A.; Singh, S. Arkivoc 2003 (xiii), 68.

29. Xu, Y.; Guo, Q. Heterocycles 2004, 63, 903.

30. Seijas, J.; Vaquez-Tato, M.; Martinez, M. Tetrahedron Lett. 2000, 41, 2215.

31. Yoon, D.; Han, Y.; Stark, J.; Haber, J.; Gregg, B.; Stankovich, S. Org. Lett. 2004, 6, 4775.

32. Scarborough, H. C.; Lawers, B. C.; Mikielli, J. L.; Compton, J. L. J. Org. Chem. 1962, 27, 957.

33. Yakhontov, L.; Zhikhareva, G.; Pronina, E.; Pershin, G.; Liberman, S.; Padeiskaya, E.; Zykova, T.; Guskova, T.; Berlyand, E. Khimiko-Farmatsevticheskii Zhurnal 1975, 9, 12.

34. Rocco, S.; Barbarini, J. E.; Rittner, R. Synthesis 2004, 3, 429. 